\title{
Production, Detection and Cross-Reactivity of Anti-Polysaccharide Antibodies from Environmental Fungi
}

\author{
Rafael Andrade Menolli ${ }^{*}$, Thais Soprani Ayala ${ }^{1}$, Pablo Rodrigo da Rosa ${ }^{1}$, \\ Claudia Rejane Lima de Macedo Costa ${ }^{2}$, Erica Fernanda Osaku ${ }^{2}$, Rosiane G. Mello Zibetti ${ }^{3}$ \\ ${ }^{1}$ Centro de Ciências Médicas e Farmacêuticas, Universidade Estadual do Oeste do Paraná, Cascavel, Brasil \\ ${ }^{2}$ Centro de Ciências Biológicas e da Saúde, Universidade Estadual do Oeste do Paraná, Cascavel, Brasil \\ ${ }^{3}$ Faculdades Pequeno Príncipe, Curitiba, Brasil \\ Email: ${ }^{*}$ rafael.menolli@unioeste.br
}

Received 13 July 2014; revised 16 August 2014; accepted 5 September 2014

Copyright (C) 2014 by authors and Scientific Research Publishing Inc.

This work is licensed under the Creative Commons Attribution International License (CC BY).

http://creativecommons.org/licenses/by/4.0/

(c) (i) Open Access

\section{Abstract}

The diagnosis of Invasive Fungal Disease (IFD) is difficult because the sensitivity of conventional culture and cytology methods for the detection of Aspergillus is poor, and because serological methods exhibit cross-reactivity with environmental fungi. Fungal polysaccharides are of particular interest in the serologic diagnosis of IFD, especially galactomannan (GM) and glucan, which have been recognized as useful tools for the diagnosis of fungal infections. Further investigation into polysaccharide antibody cross-reactivity is important in improving diagnostic tests. Our aim was to verify the production of antibodies against fungal antigens in mice immunized with exopolysaccharides (EPS) obtained from three fungal species (Aspergillus terreus, Paecilomyces variotii and Curvularia brachyspora). ELISA assays were performed to evaluate homologous and heterologous reactions and showed anti-polysaccharide antibodies production. EPS from $A$. terreus was immunogenic enough to immunize the animal and lead to antibody production against this EPS. In addition, no cross-reactivity was observed with EPS obtained from P. variotii and $C$. brachyspora. The EPS from $P$. variotii did not exhibit good immunogenicity, whereas EPS from $C$. brachyspora showed good immunogenicity, and heterologous reactions were detected on the ELISA assays performed using the EPS from this species. The results indicate that immunization can occur for environmental fungal polysaccharides, generating antibodies may be interfere in serological diagnosis that are based on detection of fungal polysaccharides, mainly glucans and galactomannans.

\section{Keywords}

Exopolysaccharides, Environmental Fungi, Immunization

\footnotetext{
${ }^{*}$ Corresponding author.
} 


\section{Introduction}

Early diagnosis and prompt introduction of antifungal therapy is essential for good prognosis in patients with systemic fungal infections or invasive fungal disease (IFD) [1]. The diagnosis of IFD is based on clinical signs assessed using radiological methods and microbiological tests, but these methodologies are not reliable and results are frequently slow to obtain, contributing to increased morbidity and mortality [2]. Invasive aspergillosis (IA) is one of the most prominent forms of IFD. This disease has been the cause of death for many individuals, with infections mainly occurring in hematopoietic stem cell transplant patients [3], in patients with certain hematologic disorders [4] and in individuals who receive organ transplants and especially lung transplants [5]. The difficulty in performing early diagnosis is one of the reasons for the high mortality because the appearance and symptoms of IA are often not specific or are attenuated by medications such as corticosteroids. In addition, the infection is generally diagnosed at advanced stages when the bioburden is high. Detection of Aspergillus spp fungus in respiratory samples is highly indicative of IA, but the culture only turns positive at the later stages of the disease [6].

The reference methodologies for IA diagnosis involve invasive procedures, as histology and culture techniques are often employed [7]. Other diagnostic tools are still under development, such as computed lung tomography and body fluid and bronchoaoveolar lavage fluid cultures, which exhibit low sensitivity. Recent research efforts have focused on methods not based on cultures or invasive procedures [1].

The main necessary characteristics of a routine diagnostic test include reliability and reproducibility, crosslaboratory standardization, ease of administration, speed of results and financial applicability [2].

Laboratory diagnoses of fungal infections are tricky due to the lack of sensitivity and/or specificity of cultures. As mentioned, the use of non culture-based methods in the management of patients with risk of IFD is rising. One alternative is the use of available biomarkers, fungal antigens and molecular tests that might improve premature diagnosis. Biomarkers are already used in patients with malignant hematologic disorders during screening and selection of antifungal treatment [2]. A revised version of the European Organization for the Research and Treatment of Cancer/Mycoses Study Group includes biomarkers such as galactomannan (GM), $\beta$-glucan and Cryptococcus antigen [8].

Fungal carbohydrates can induce antibody production that increases host defense and resistance in many ways. Fueled by advances in molecular and cellular biology, a high number of fungal proteins and carbohydrates that initiate T cell immunity and that are immunogenic in mouse models of fungal infections have been identified [9]. The US Food and Drug Administration (FDA), an American regulatory authority, approved in 2003 a serologic assay utilizing the enzyme-linked immunosorbent assay (ELISA) technique to detect glycoproteins known as galactomannans (GM), which are present in the cell wall of Aspergillus. For common microorganisms, methods that demonstrate the presence or allow for the isolation of the causative agent are sufficient because they exhibit good sensitivity. However, in the case of opportunistic microorganisms, emergent pathogens or pathogens with restricted growth, these methods are not appropriate, and it is necessary to establish differential diagnostic methods such as serologic assays. In the past few years, research has focused on the search for antibodies and antigens in the serum of patients. Antibodies and antigens have been shown to be of value in the diagnosis, prognosis and monitoring of antifungal therapies [1].

A diagnostic test for fungal diseases should perform adequately, ensuring a minimum level of certainty with regard to the presence or absence of disease. Tests must also show positive results even in the early stages of the disease [10]. Until now, no method has shown enough sensitivity and specificity to allow for reliable early diagnosis of fungal infections. Accordingly, investigation into new methods and techniques is necessary to satisfy clinical practice and routine [7].

\section{Materials and Methods}

\subsection{Polysaccharides Characterization}

Exopolysaccharides (EPS) from three anemophylous fungi (Aspergillus terreus, Curvularia brachyspora and Paecilomyces variotii) were used as immunogens and obtained from optimized submerged cultures. After growth, the media was harvested and filtered, and polysaccharides were precipitated with ethanol, dialyzed and lyophilized. Cold water-insoluble materials were eliminated by centrifugation, the soluble materials were treated with amylase. Polysaccharide homogeneity and molar mass determination were determined by high performance 
steric exclusion chromatography with multi-angle laser light scattering (HPSEC-MALLS), using a refractive index (RI) detector. The specific refractive index increment $(\mathrm{dn} / \mathrm{dc})$ was determined using a Waters 2410 detector. The molecular weight were performed by the Astra 4.72 software (Wyatt Technology). Monosaccharide compositions were performed by the alditol acetate method [11] with minor modifications. Firstly, the polysaccharide was hydrolyzed by $2 \mathrm{M} \mathrm{TFA}$ at $100^{\circ} \mathrm{C}$ for $8 \mathrm{~h}$, followed by reduction in distilled water with $\mathrm{NaBH}_{4}$ and acetylated with 1:1 $\mathrm{Ac}_{2} \mathrm{O}$-pyridine $(2 \mathrm{~mL})$ at room temperature for $12 \mathrm{~h}$. The resulting alditol acetates were analyzed by GC-MS. ${ }^{13} \mathrm{C}$-NMR was carried out using a $400 \mathrm{MHz}$ Bruker model DRX Advance spectrometer incorporating a Fourier transform. The samples were dissolved in $\mathrm{Me}_{2} \mathrm{SO}-\mathrm{d}_{6}$ and examined at 50 or $70^{\circ} \mathrm{C}$. The chemical shifts of the samples are expressed in ppm $(\delta)$ relative to the resonance of $\mathrm{Me}_{2} \mathrm{SO}-\mathrm{d}_{6}$ at $\delta 37.70\left({ }^{13} \mathrm{C}\right)$. The three EPS were preliminarily characterized as $\beta$-Glucan $(1 \rightarrow 3)$ (P. variotii), Glucogalactomannan (A. terreus) and Galactomannan (C. brachyspora).

\subsection{Mouse Immunization and Serum Collections}

For immunization, one milligram of each EPS was inoculated either alone or emulsified in an equal volume of Freund's adjuvant. Thirty-five C57BL/6 mice (8 weeks old) were separated into seven groups, consisting of a negative control group (immunized with sterile saline) and six experimental groups immunized with each of the three EPS, either alone or in combination with Freund's adjuvant. The immunization was performed via intradermic inoculation. Animals were primed with complete adjuvant on the first day and were given two boosters with incomplete adjuvant on the tenth and twentieth days. Mice were bled twenty days after the last booster. Two independent experiments were performed for each group. All legal recommendations from the Brazilian legislation (Law No. 11.794/October 2008) for animal handling procedures in scientific research were followed, and all animal procedures were approved by the Animal Ethics Committee of the State University of Western Parana (UNIOESTE).

\subsection{Enzyme-Linked Immunoassay}

We developed an enzyme-linked immunoassay (ELISA) to detect antibodies generated by immunizations and any possible cross-reactivity between different antibodies generated by the different antigens utilized. First, a sensitization experiment was performed in a 96-well plate using various EPS concentrations from the three fungi to standardize the optimal concentration for further experiments. EPS (1 mg) was dissolved in $1 \mathrm{ml}$ of a PBS solution containing $0.02 \%$ sodium a zide and diluted to final concentrations of $50 \mu \mathrm{g} / \mathrm{ml}, 25 \mu \mathrm{g} / \mathrm{ml}, 10 \mu \mathrm{g} / \mathrm{ml}$ and $5 \mu \mathrm{g} / \mathrm{ml}$. Diluted EPS was added to 96-well plates at $100 \mu \mathrm{L}$ per well and subjected to an ELISA assay against non-diluted sera obtained from immunized mice.

An indirect ELISA was established using EPS as the antigen and HRP-anti-mouse IgG as the conjugate to detect antibodies produced in animals. After standardizing the optimal antigen concentration, sera were tested using an ELISA against the EPS used for immunization to detect homologous reactions indicating the presence of anti-polysaccharide antibodies. Sera were also tested using the other two EPS to detect heterologous reactions indicating the occurrence of cross-reactivity.

\subsection{Statistical Analysis}

Serum samples were analyzed in quadruplicate, and data were statistically evaluated using variance analysis (oneway ANOVA) and Tukey's test for comparison of averages. Data are reported as the mean values \pm S.D, and values were considered significant when $p<0.05$.

\section{Results and Discussion}

Prior to performing the ELISA assays, the adequate antigen concentration for optimal sensitivity in the ELISA reactions was determined. Test reactions were setup to identify the best standard concentration. The best concentrations according to the test results are listed in Table 1.

The standard EPS concentrations for A. terreus, P. variotii and C. brachyspora fungi were decided based on the absorbance measured in tests in which sera from immunized animals were challenged with different antigen concentrations. Out of the reactions that were not significantly different from the absorbance values observed in the reaction between pure sera and the different concentrations of EPS tested, we chose the concentration that 
Table 1. Fungal EPS concentrations standardized for use in indirect ELISA.

\begin{tabular}{cc}
\hline EPS Species & Concentration \\
\hline Aspergillus terreus & $10 \mu \mathrm{g} / \mathrm{ml}$ \\
Paecilomyces variotii & $25 \mu \mathrm{g} / \mathrm{ml}$ \\
Curvularia brachyspora & $25 \mu \mathrm{g} / \mathrm{ml}$ \\
\hline
\end{tabular}

resulted in the highest absorption and the lowest variation between replicates, indicating optimal performance. ELISA plates were sensitized with the optimal antigen concentrations to investigate possible homologous and heterologous reactions, making it possible to verify whether animals previously immunized with EPS from one fungal species produced antibodies against that same EPS or whether animals produced antibodies that are able to complex with EPS from other fungi.

Other works that aimed to establish an immunoenzimatic assay for detection of fungal anti-polysaccharide antibodies by own methodologies [11] [12] have used concentrations close to the used in this research. These concentrations appear to be high for clinical and laboratorial use, but seem to be interesting to determine if indeed it is possible to obtain antibodies from fungal polysaccharides. This implies that the commercial use of these antigens should be further assessed.

Figure 1 shows that the animals were able to respond to the EPS from A. terreus and that this immunization occurred with and without adjuvant, although higher $(p<0.01)$ absorbances were observed in the group with added adjuvant. There were significant differences between the absorbance values obtained from animals immunized with A. terreus EPS, with or without adjuvant, and the negative control $(p<0.001)$, and these values were significantly higher than those obtained in heterologous reactions. High absorbances in heterologous reactions were mainly observed when sera of animals immunized with EPS from P. variotii and C. brachyspora, with or without adjuvant, were challenged with $A$. terreus antigen. No differences were observed between results from heterologous groups and the control (non-immunized animals).

The results from the reaction of the A. terreus EPS antigen with sera from animals immunized with EPS from $P$. variotii and $C$. brachyspora imply a low level of cross-reactivity between the antibodies produced by these animals against $A$. terreus EPS. These data are important because they present the possibility that this test is relatively specific.

According to the data we obtained with the immunization using EPS from P. variotii, the animals did not respond satisfactorily, based on the low absorbance observed in the homologous reactions to this antigen (Figure 2). There was a significant difference between the control group and the group immunized with EPS from P. variotii along with adjuvant. Analyzing the groups immunized with EPS from P. variotii, C. brachyspora and C. brachyspora with adjuvant, there were no differences between these groups. In addition, there was a significant difference between the group immunized with $A$. terreus EPS plus adjuvant compared with the other groups.

These results show that the $P$. variotii EPS has a low immunogenic capacity and that this antigen may not be appropriate to be used in diagnostic tests for infections by this fungus. A better antigen from this fungus will need to be identified. There was no apparent cross-reactivity observed with the groups immunized with $C$. brachyspora EPS with or without adjuvant, but cross-reactivity signals were observed between sera obtained from A. terreus with or without adjuvant against $P$. variotii EPS. This could be due to the high titers obtained upon immunization with $A$. terreus, which could be interfering as a false positive against other antigens.

Figure 3 shows data obtained from immunization of mice with EPS from C. brachyspora. Animals exhibited good immunogenic responses to this antigen, with no difference occurring between the groups with and without adjuvant. Significant differences $(p<0.05)$ were observed between the control group and the groups immunized with EPS from C. brachyspora, either with or without the adjuvant. As such, significant differences could be observed between the control group and the group immunized with EPS from $P$. variotii, $P$. variotti with added adjuvant, and $A$. terreus with added adjuvant. No differences were observed between the control group and the group immunized with EPS from A. terreus.

Additionally, significant differences were not observed between the group immunized with $C$. brachyspora and the groups setup as heterologous reactions on the plate; i.e., the groups immunized with EPS from $P$. variotii, $P$. variotii with added adjuvant and $A$. terreus with added adjuvant. These data indicate the occurrence of significant cross-reactivity, which decreases the specificity of this assay.

The results obtained with EPS from C. brachyspora showed that this antigen has good immunogenicity, high- 


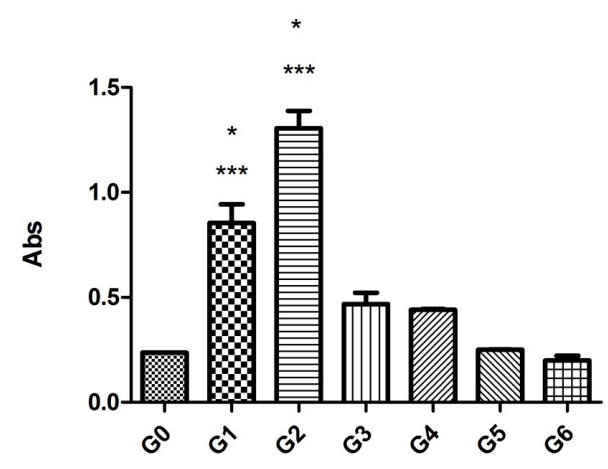

Figure 1. Homologous and heterologous reaction in 96-well plates sensitized with $10 \mu \mathrm{g} / \mathrm{ml}$ EPS from A. terreus. G0, control group; G1, group immunized with EPS from A. terreus; G2, group immunized with EPS from A. terreus containing added adjuvant (G1 and G2 are designed to detect homologous reactions); G3, group immunized with EPS from $P$. variotii; G4, group immunized with EPS from $P$. varioiticontaining added adjuvant; G5, group immunized with EPS from $C$. brachyspora; G6, group immunized with EPS from C. brachyspora added adjuvant $\left({ }^{* * *} p<0.001\right.$ compared with G0; ${ }^{*} p<$ 0.05 compared with G3, G4, G5 and G6).

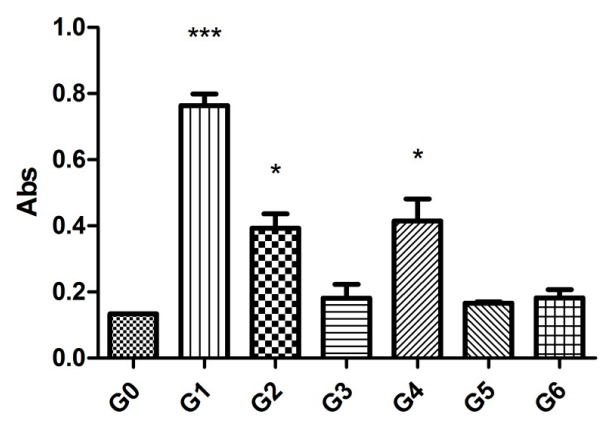

Figure 2. Homologous and heterologous reaction in 96-well plates sensitized with $25 \mu \mathrm{g} / \mathrm{mL}$ EPS from P. variotii. G0, control group; G3, group immunized with EPS from P. variotii; G4, group immunized with EPS from $P$. variotti added adjuvant (G3-G4 are designed to detect homologous reactions); G2, group immunized with EPS from A. terreus; G1, group immunized with EPS from A. terreus added adjuvant; G5, group immunized with EPS from C. brachyspora; G6, group immunized with EPS from $C$. brachyspora added adjuvant (G1, G2, G5 and G6 are designed to detect heterologous reaction). $\left({ }^{* * *} p<0.001\right.$ compared with G0, G3, G5 and G6; ${ }^{*} p<0.05$ compared with G0, G3, G5 and G6).

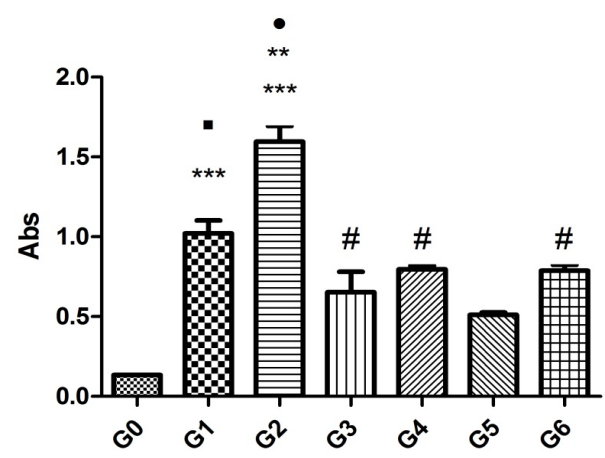

Figure 3. Homologous and heterologous reactions in 96-well plate sensitized with $25 \mu \mathrm{g} / \mathrm{ml}$ EPS from C. brachyspora. G0, control group; G1, group immunized with EPS from C. brachyspora; G2, group immunized with EPS from C. brachyspora added adjuvant (G1 and G2 are designed to detect homologous reactions); G3, group immunized with EPS from P. variotii; G4 group immunized with EPS from P. variotiiadded adjuvant; G5, group immunized with EPS from A. terreus; G6, group immunized with EPS from $A$. terreus added adjuvant $\left({ }^{* * * *} p<0.001\right.$ compared with $\mathrm{G} 0 ;{ }^{* *} p<0.01$ compared with G1; $p<$ 0.001 compared with G3, G4, G5 and G6; $p<<0.05$ compared with G5; \#p < 0.05 compared with G0).

lighting the importance of establishing how anti-polysaccharide antibodies from C. brachyspora may interfere with serologic reactions, due to the high prevalence of $C$. brachyspora in the environment and the possible sen- 
sitization of individuals to the polysaccharide of this fungus and consequent antibody production against this antigen [12].

Fungal exopolysaccharides, with external distribution on fungal cell wall, are main contact with mammalian immune cells [13], and, since many years ago, are more important component in field of searching new targets for serologic diagnosis. Others fungal components, as DNA, have similar performance with serologic tests [14], while fungal proteins have been used for allergenic detections [15].

Currently, there are few serologic test options for infections caused by fungi, and these tests aim to detect certain key fungal polysaccharides, such as galactomannan and glucan. However, to detect antigens, a large microbial burden is usually necessary, which is a limitation of these tests [16]. The sensitivity of these tests (approximately $70 \%$ ) is similar to that of other methodologies (cultures or imaging) [17], which generates some difficulty in confirming a diagnosis using this assay because even prophylactic treatments or anti-galactomannananti body production from an Aspergillus exposure may result in false negative results [18]. Thus, antibodies generated by contact with polysaccharides from environmental fungi belonging to others genera could have the same effect, once our data showed cross-reactivity between in so different fungi.

Specificity is of high interest in serological diagnostic testing because one of major problems for immunocompromised patients who may develop aspergillosis is a previous sensitization by environmental fungi, mostly due to allergic reactions. Once sensitized by this environmental fungi, antibody production against the environmental antigen can occur and cause cross-reactivity. Faster, higher-quality and more exact diagnoses can be developed when cross-reactivity is low. One of the obstacles to establishing a diagnostic test for fungal infections based on immunological reactions is the occurrence of cross-reactivity with a lack of specificity, as there are many environmental fungi that individuals can come in contact with and become sensitized to, leading to the production of antibodies that may easily cross-react with other fungal epitopes, invalidating most immunological tests and generating many false-positive results [2]. False-positive results could be generated by antibodies generated by fungal polysaccharides isolated from environmental fungi, which are able to stimulate immune system of exposed individuals, what may explain why the combination of tests using different antigensare capable of increasing the sensitivity and false positives results [17]. Search for better specificity, without lost of sensibility, is the eventual goal of searching for new diagnostic tests for IFD to patients with immunological limitations [14].

Most work to approach the production of antibodies to environmental fungal polysaccharides were focused on the production of IgE [19], because the importance the seantigens in allergies. Production of IgG is few explored and the standardization of this detection proves are complicated in in-house kits produced for this purpose [20]. Is important to note that a constant contact with environmental fungi, such as $P$. variotti and $C$. brachyspora are easily found on poultry farms [21] [22], and the immunization eventually could occur.

Some studies have shown [23] that the use of GM detection as a screening test when monitoring transplant patients, for example, can be a great ally in the early detection of IFD, reducing the use of empirical antifungal treatment and improving the clinical management of patients, mainly the high-risk groups. Moreover, in that study, no positive cases were missed when biomarker-based diagnostic tests were used [23] and, more cases of IA were identified using diagnostic tests based on biomarkers than based on standard methods. The biomarkers in this study showed improvement, suggesting that a diagnosis based on biomarkers can be used as tool in routine diagnosis, improving differentiation between patients positive or negative for IA and helping doctors make practical decisions. In addition, these results show that standard methods under-diagnose IA [24].

There have been reported difficulties in serologically diagnosing certain opportunistic fungal infections such as blastomycosis, for which cross-reactivity is the critical barrier to diagnosis using antibodies because antibodies have been shown to cross-react with the fungi that cause coccidiodomycosis, histoplasmosis, paracoccidioidomycosis and other types of infections [16]. Another pathology that has been the focus of research is coccidioidomycosis, a disease for which the serologic diagnosis is built on the detection of an antibody against two antigens from this fungus [25]. One of the antigens is associated with the acute phase of the disease and the other one is associated with the chronic phase of the disease. However, there is a limitation because these antibodies may not be present in immune compromised patients [16]. For histoplasmosis the diagnosis is based mainly on isolation and culture for identification of the fungus; nevertheless, results take between 2 - 4 weeks, whereas an identification of antibodies against the fungus can provide a more rapid diagnostic alternative compared with the microbiological technique [16]. In the diagnosis of paracoccdioidomycosis, the detection of an anti-P. brasiliensis antibody is already used when IgG levels are high in patients with the disease [6]. These antibodies are also used 
to monitor the effect of treatments and the evolution of the disease.

Because it does not require an invasive procedure, the detection of a specific host-antibody response is a pathway to a successful diagnosis. Unfortunately, mainly in immunocompromised patients, the presence of antibodies resulting from stimulation by the presence of the pathogen does not always correlate with an invasive disease [16]. In addition, antibody detection can serve as a supplementary diagnosis. Cross-reaction between species, the presence of antibodies against environmental fungi and the absence of sensitivity are some of the challenges that need to be overcome in the diagnosis of fungal infections [16].

There are few studies demonstrating the ability of polysaccharides from environmental fungi in generating antibodies from animal models, and this work characterized the production of anti-fungal polysaccharide IgG antibodies. These polysaccharides are in the environment and may sensitize the immune system of individuals that, once exposed to systemic fungal infections, may present interference to the serological tests for these infections. Future studies in our laboratory will define the ability to generate anti-polysaccharide antibodies in experimental fungal infections promoted by environmental fungi.

\section{References}

[1] Florent, M., Katsahian, S., Vekhoff, A., Levy, V., Rio, B., Marie, J, et al. (2006) Prospective Evaluation of a Polymerase Chain Reaction-ELISA Targeted to Aspergillus fumigatus and Aspergillus flavus for the Early Diagnosis of Invasive Aspergillosis in Patients with Hematological Malignancies. The Journal of Infectious Disease, 193, 741-747. http://dx.doi.org/10.1086/500466

[2] Marchetti, O., Lamoth, F., Mikulska, M., Viscoli, C., Verweij, P. and Bretagne, S. (2012) ECIL Recommendations for the Use of Biological Markers for the Diagnosis of Invasive Fungal Diseases in Leukemic Patients and Hematopoietic SCT Recipients. Bone Marrow Transplantation, 47, 846-854. http://dx.doi.org/10.1038/bmt.2011.178

[3] Fukuda, T., Boeckh, M., Carter, R.A., Sandmaier, B.M., Maris, M.B., Maloney, D.G., et al. (2003) Risks and Outcomes of Invasive Fungal Infections in Recipients of Allogeneic Hematopoietic Stem Cell Transplants after Nonmyeloablative Conditioning. Blood, 102, 827-833. http://dx.doi.org/10.1182/blood-2003-02-0456

[4] Martino, R. and Subirà, M. (2002) Invasive Fungal Infections in Hematology: New Trends. Annals of Hematology, 81, 233-243. http://dx.doi.org/10.1007/s00277-002-0466-3

[5] Singh, N. and Husain, S. (2003) Aspergillus Infections after Lung Transplantation: Clinical Differences in Type of Transplant and Implications for Management. The Journal of Heart and Lung Transplantation, 22, 258-266. http://dx.doi.org/10.1016/S1053-2498(02)00477-1

[6] Pound, M.W., Drew, R.H. and Perfect, J.R. (2002) Recent Advances in the Epidemiology, Prevention, Diagnosis, and Treatment of Fungal Pneumonia. Current Opinion in Infectious Diseases, 15, 183-194. http://dx.doi.org/10.1097/00001432-200204000-00014

[7] Ascioglu, S., Rex, J.H., De Pauw, B., Bennett, J.E., Bille, J., Crokaert, F., et al. (2002) Defining Opportunistic Invasive Fungal Infections in Immunocompromised Patients with Cancer and Hematopoietic Stem Cell Transplants: An International Consensus. Clinical Infectious Diseases, 34, 7-14. http://dx.doi.org/10.1086/323335

[8] De Pauw, B., Walsha, T.J., Donnellya, J.P., Stevens, D.A., Edwards, J.E., Calandra, T., et al. (2008) Revised Definitions of Invasive Fungal Disease from the European Organization for Research and Treatment of Cancer/Invasive. Clinical Infectious Diseases, 46, 1813-1821. http://dx.doi.org/10.1086/588660

[9] Cutler, J.E., Deepe, G.S. and Klein, B.S. (2007) Advances in Combating Fungal Diseases: Vaccines on the Threshold. Nature Reviews Microbiology, 5, 13-28. http://dx.doi.org/10.1038/nrmicro1537

[10] Quindo, G. (2006) New Microbiological Techniques for the Diagnosis of Invasive Mycoses Caused by Filamentous Fungi. Clinical Microbiology and Infection, 12, 40-52. http://dx.doi.org/10.1111/j.1469-0691.2006.01605.x

[11] Crowell, E.P. and Burnett, B.B. (1967) Determination of the Carbohydrate Composition of Wood Pulps by Gas Chromatography of the Alditol Acetates. Analytical Chemistry, 39, 121-124. http://dx.doi.org/10.1021/ac60245a006

[12] Cavanna, C., Seminari, E., Pusateri, A., Mangione, F., Lallitto, F., Esposto, M.C., et al. (2014) Allergic Fungal Rhinosinusitis Due to Curvularia lunata. New Microbiologica, 37, 241-245.

[13] Latgé, J.P. (2010) Tasting the Fungal Cell Wall. Cellular Microbiology, 12, 863-872. http://dx.doi.org/10.1111/j.1462-5822.2010.01474.X

[14] Kawazu, M., Kanda, Y., Nannya, Y., Aoki, K., Kurokawa, M., Chiba, S., et al. (2004) Prospective Comparison of the Diagnostic Potential of Real-Time PCR, Double-Sandwich Enzyme-Linked Immunosorbent Assay for Galactomannan, and a (1 3)- $\beta$-D-Glucan Test in Weekly Screening for Invasive Aspergillosis in Patients with Hematological Disorders. Journal of Clinical Microbiology, 42, 2733-2741. http://dx.doi.org/10.1128/JCM.42.6.2733-2741.2004

[15] Zahradnik, E., Kespohl, S., Sander, I., Schies, U., Khosravie-Hohn, J., Lorenz, W., et al. (2013) A New Immunoassay 
to Quantify Fungal Antigens from the Indoor Mould Aspergillus versicolor. Environmental Science: Processes \& Impacts, 15, 1162-1171. http://dx.doi.org/10.1039/c3em30870g

[16] Yeo, S.F. and Wong, B. (2002) Current Status of Nonculture Methods for Diagnosis of Invasive Fungal Infections. Clinical Microbiology Reviews, 15, 465-484. http://dx.doi.org/10.1128/CMR.15.3.465

[17] Pfeiffer, C.D., Fine, J.P. and Safdar, N. (2006) Diagnosis of Invasive Aspergillosis Using a Galactomannan Assay: A Meta-Analysis. Clinical Infectious Diseases, 42, 1417-1427.

[18] Wheat, L.J. and Walsh, T.J. (2008) Diagnosis of Invasive Aspergillosis by Galactomannan Antigenemia Detection Using an Enzyme Immunoassay. European Journal of Clinical Microbiology \& Infectious Diseases, 27, 245-251. http://dx.doi.org/10.1007/s10096-007-0437-7

[19] Douwes, J., Van Der Sluis, B., Doekes, G., Van, F., Wijnands, L., Van Strien, R., et al. (1999) Fungal Extracellular Polysaccharides in House Dust as a Marker for Exposure to Fungi: Relations with Culturable Fungi, Reported Home Dampness, and Respiratory Symptoms. Environmental and Occupational Disorders, 103, 494-500.

[20] Barton, R.C., Hobson, R.P., Denton, M., Peckham, D., Brownlee, K., Conway, S., et al. (2008) Serologic Diagnosis of Allergic Bronchopulmonary Aspergillosis in Patients with Cystic Fibrosis through the Detection of Immunoglobulin G to Aspergillus fumigatus. Diagnostic Microbiology and Infectious Disease, 62, 287-291. http://dx.doi.org/10.1016/j.diagmicrobio.2008.06.018

[21] Taira, C.L., Marcondes, N.R. and Mota, V.A. (2011) Virulence Potential of Filamentous Fungi Isolated from Poultry Barns in Cascavel, Paraná, Brazil. Brazilian Journal of Pharmaceutical Sciences, 47, 155-160.

[22] Marcondes, N.R., Taira, C.L., Vandresen, D.C., Svidzinski, T.I.E., Kadowaki, M.K. and Peralta, R.M. (2008) New Feather-Degrading Filamentous Fungi. Microbial Ecology, 56, 13-17. http://dx.doi.org/10.1007/s00248-007-9319-x

[23] Maertens, J., Theunissen, K., Lodewyck, T., Lagrou, K. and Van Eldere, J. (2007) Advances in the Serological Diagnosis of Invasive Aspergillus Infections in Patients with Haematological Disorders. Mycoses, 50, 2-17. http://Dx.Doi.Org/10.1111/J.1439-0507.2007.01375.X

[24] Morrissey, C.O., Chen, S.C., Sorrell, T.C., Milliken, S., Bardy, P.G., Bradstock, K.F., et al. (2013) Galactomannan and PCR versus Culture and Histology for Directing Use of Antifungal Treatment for Invasive Aspergillosis in High-Risk Haematology Patients: A Randomised Controlled Trial. The Lancet Infectious Diseases, 13, 519-528. http://dx.doi.org/10.1016/S1473-3099(13)70076-8

[25] Blair, J.E., Coakley, B., Santelli, A.C., Hentz, J.G. and Wengenack, N.L. (2006) Serologic Testing for Symptomatic Coccidioidomycosis in Immunocompetent and Immunosuppressed Hosts. Mycopathologia, 162, 317-324. http://dx.doi.org/10.1007/s11046-006-0062-5. 
Scientific Research Publishing (SCIRP) is one of the largest Open Access journal publishers. It is currently publishing more than 200 open access, online, peer-reviewed journals covering a wide range of academic disciplines. SCIRP serves the worldwide academic communities and contributes to the progress and application of science with its publication.

Other selected journals from SCIRP are listed as below. Submit your manuscript to us via either submit@scirp.org or Online Submission Portal.
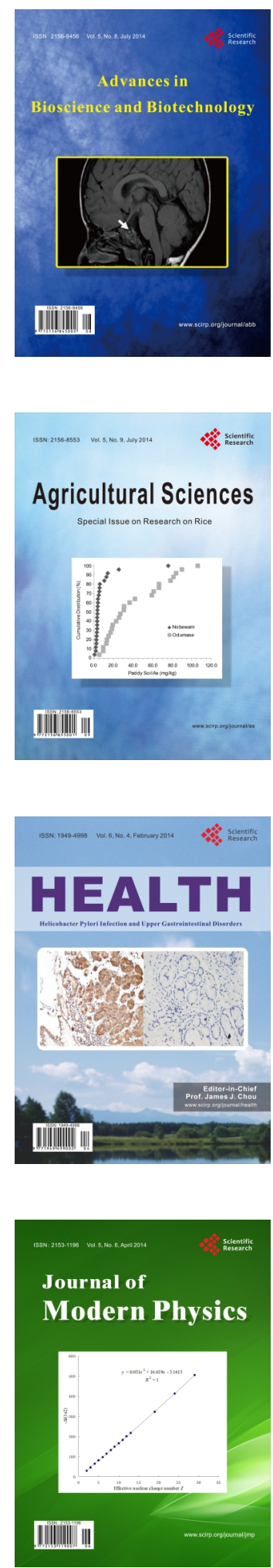
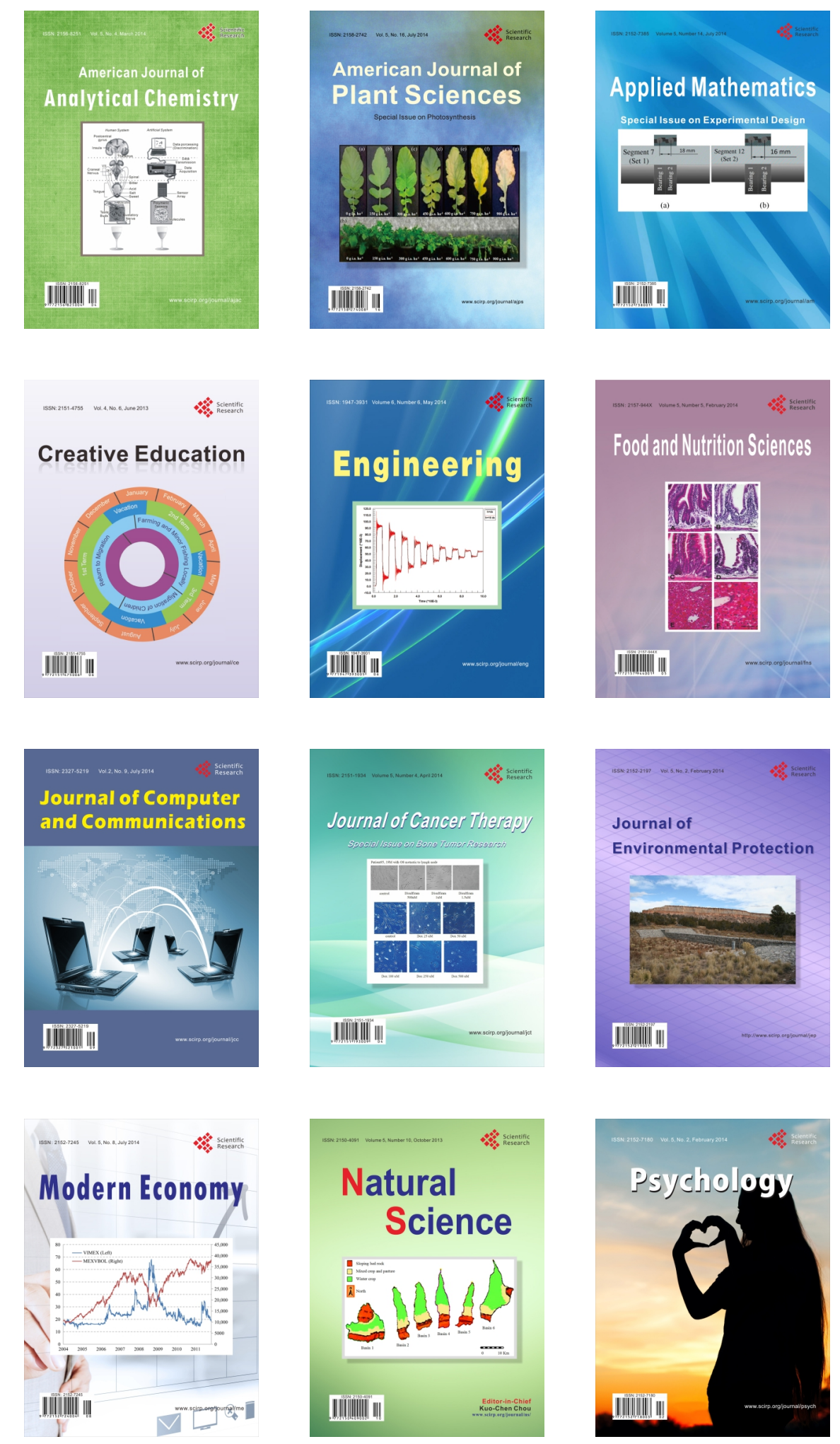